\title{
Ocular Demodex folliculorum: prevalence and associated symptoms in an Irish population
}

\author{
Orla Murphy \\ Technological University Dublin, orla.murphy@tudublin.ie \\ Veronica O'Dwyer \\ Technological University Dublin, veronica.odwyer@tudublin.ie \\ Aoife Lloyd-Mckernan \\ Technological University Dublin, aoife.lloydmckernan@tudublin.ie
}

Follow this and additional works at: https://arrow.tudublin.ie/scschphyart

Part of the Ophthalmology Commons, and the Optometry Commons

\section{Recommended Citation}

Murphy, O., O'Dwyer, V. \& Lloyd-McKernan, A. Ocular Demodex folliculorum: prevalence and associated symptoms in an Irish population. Int Ophthalmol 39, 405-417 (2019). DOI: 10.1007/s10792-018-0826-1

This Article is brought to you for free and open access by the School of Physics \& Clinical \& Optometric Science at ARROW@TU Dublin. It has been accepted for inclusion in Articles by an authorized administrator of ARROW@TU Dublin. For more information, please contact arrow.admin@tudublin.ie, aisling.coyne@tudublin.ie, gerard.connolly@tudublin.ie.

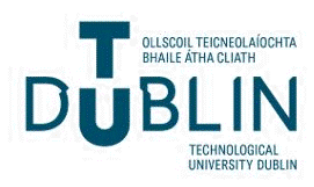




\title{
Ocular Demodex folliculorum: prevalence and associated symptoms in an Irish population
}

\author{
Orla Murphy (D) - Veronica O’Dwyer • Aoife Lloyd-McKernan
}

Received: 7 April 2017 / Accepted: 19 January 2018/Published online: 29 January 2018

(C) Springer Science+Business Media B.V., part of Springer Nature 2018

\begin{abstract}
Purpose To investigate the prevalence of ocular Demodex folliculorum (DF) in an Irish population. To validate a modified Ocular Surface Disease Index questionnaire and employ it to evaluate the relationship between dry eye symptoms and the presence of DF.

Methods One hundred and fifty-six subjects were enrolled in an epidemiological cross-sectional prevalence study. Each subject completed a novel questionnaire on ocular symptoms and was assessed for the presence of DF. Data was analysed to assess prevalence and to search for significant links between each symptom and DF.

Results An overall prevalence of $68 \%$ was found. Total mean number of DF found on microscopic examination was 3.83 mites per subject (range 0-25). The presence of symptoms was higher among individuals with $\mathrm{DF}(P=0.04)$. Itch was found to be the symptom most significantly associated with the presence and number of DF $(P=0.025$ and $P=0.035$, respectively). The questionnaire showed good internal
\end{abstract}

Electronic supplementary material The online version of this article (https://doi.org/10.1007/s10792-018-0826-1) contains supplementary material, which is available to authorized users.

O. Murphy $(\bowtie) \cdot$ V. O’Dwyer · A. Lloyd-McKernan The National Optometry Centre, Dublin Institute of Technology, Kevin Street, Dublin 8, Ireland e-mail: orla.murphy@dit.ie consistency (Cronbach's alpha; $\alpha>0.7$ ) and good reliability (Intra-class Correlation Co-efficient; ICC > 0.7). The sensitivity and specificity of a positive symptom result using the questionnaire were $70.8 \%$ and $46.9 \%$, respectively.

Conclusion There is a strong association between the prevalence of DF and symptoms, in particular itchy eyes. However, not all patients with DF will be symptomatic. The newly developed questionnaire is a reliable instrument for measuring change in symptoms over a period of time and suitable for observing patient reported outcomes in interventional treatment studies.

Keywords Demodex folliculorum - Prevalence - Dry eye symptoms · Blepharitis

\section{Introduction}

Demodex folliculorum (DF) and Demodex brevis (DB) are eight legged ectoparasites that reside in the pilosebaceous units of human skin [1-5]. The mites feed on epidermal skin cells and sebum and are, therefore, most commonly found in areas rich in sebaceous glands-cheeks, nose, chin and the periocular area [2, 4-6]. DF inhabits the eyelash follicles. It uses its claws to scrape at the internal walls of the lash follicles resulting in follicular distention, epithelial hyperplasia and reactive hyperkeratinisation 
$[3,5,7,8]$. It is suggested that this forms a translucent cuff at the base of the lash [9]: cylindrical dandruff (CD). It has also been proposed that CD is the result of an inflammatory cicatrix formed from dead DF within the follicle [10]. CD is considered a pathognomonic sign for DF infestation of the eyelash follicles [11]. Studies suggest DB resides in the sebaceous glands and contributes to meibomian gland dysfunction (MGD) by causing granulomatous changes to the glandular cells and also physically blocking the gland resulting in an accumulation of meibum within the duct $[3,5,8]$. Given the location of the mites on the eyelids; DF appears to be associated with anterior blepharitis and DB with MGD. The resulting eyelid inflammation causes inflammatory proteins to be released into the tear film causing ocular surface inflammation and irritation $[12,13]$.

Various ocular abnormalities such as anterior blepharitis, MGD, aqueous deficient and evaporative dry eye share similar symptoms involving the ocular surface; itch, irritation, redness, burning sensations $[7,14,15]$. This creates a challenge for practitioners to distinguish between each condition when screening patients based on symptoms alone.

Another dilemma is that dry eye is a multifactorial disease, and the symptoms of dry eye and ocular surface disease fluctuate and often do not correlate well with the degree of ocular signs present [16-19]. Symptoms and severity of symptoms may vary according to the condition and time of day [20]. MGD and blepharitis are more commonly associated with a foreign body sensation and sticky eyes in the morning, while aqueous deficient dry eye appears to get worse throughout the day [20]. DF and DB can produce an inflammatory reaction on the ocular surface [21]. Itching and crusting at the eyelid margin are among the most common symptoms associated with DF infestation [7, 15, 22, 23]. This is most likely due to the presence of $\mathrm{CD}$ on the eyelash margin and the movement of the mites across the surface of the skin. As mites are most active at night [24], it could be hypothesised that patients with DF infestation would be more symptomatic at night or in the morning after the mites have been most active. To try to better understand the relationship between DF and symptoms, it is important for subjects to report on the frequency and severity of them. This also applies to functional vision such as reading, using a computer, and environmental conditions known to exacerbate symptoms in certain ocular surface diseases, for example, wind and air conditioning [20, 25].

Increasingly, patient reported outcomes (PROs) are becoming an integral part of clinical trials [26, 27]. Monitoring PROs after treatment is a good way of investigating the effect of treatment on the patient [27]. In 2011, the International Workshop on Meibomian Gland Dysfunction discussed the increasing importance of PROs in clinical trials. A recommendation was also made to attempt to identify specific symptoms for specific conditions, as the difficulty in distinguishing between symptoms of different anterior abnormalities is an ever present challenge [28]. The present questionnaire was developed and analysed to assess the relationship between DF and dry eye symptoms. The capacity for the questionnaire to be used as a diagnostic screener for DF blepharitis and its ability to assess change in symptoms over the course of treatment was also evaluated.

\section{Materials and methods}

\section{Ethics statement}

This study was conducted under the Tenets of Helsinki Declaration of Human Studies [29] after approval by the Dublin Institute of Technology Research Ethics Committee.

\section{Examination}

Subjects from the National Optometry Centre, local catchment area, and staff and students of Dublin Institute of Technology were invited to part-take in a cross-sectional prevalence study for ocular DF. Inclusion criteria: $\geq 18$ years of age. Exclusion criteria: subjects currently being treated for blepharitis or who had used treatment in the past 6 months, active ocular infection (excluding blepharitis) or ocular surgery within the past 6 months. Each subject completed the novel questionnaire and was assessed for the presence of DF. Presence of DF was defined as: positive sighting of DF on lash rotation and/or one or more DF counted on microscopic examination. One eyelash from each eyelid was first manipulated in clockwise and counter-clockwise directions using a sterile forceps, to stimulate DF tails, if present, to emerge from the lash follicle. Subsequently, the lash was epilated 
for microscopic examination. Currently, rotation of the eyelash prior to epilation is not a standardised technique. However, its use in visualising DF prior to epilation has been utilised in previous studies [5, 30]. Furthermore, it has been highlighted that lash manipulation has the potential to provide an alternative method to lash epilation for visualising DF in a clinical setting, although further research is required at present $[31,32]$. Thus, epilation and microscopic examination remain the recommended method for DF diagnosis [7, 32]. Adult DF count was recorded using the modified Coston method [11]. Data was analysed to assess the overall prevalence of DF found, and to search for significant links between each symptom and the presence and quantity of DF.

\section{Questionnaire development}

The symptom questionnaire was styled on the validated OSDI symptom questionnaire (Attachment 1). It has been suggested that altering an existing questionnaire may help increase sensitivity of the symptom questionnaire for the diagnosis of MGD or blepharitis [33]. The OSDI format was chosen, as it is has shown good repeatability and validity for effectively measuring the severity of dry eye [34]. It is one of the most commonly used symptom questionnaires administered to subjects in DF related clinical trials $[15,35,36]$, and the scoring system utilised by OSDI is scored on scale of 0-100 with higher scores indicating increased severity of symptoms. Each item in the questionnaire is graded on a 4-point Likert scale indicating frequency of the symptom in question; where 0 equals none of the time, 1 -some of the time, 2-half of the time, 3-most of the time, and 4-all of the time. The total OSDI score is calculated using the following formula: [total symptom number $(A) \times 25 /$ number of questions answered (B)] [34, 37]. As the formula takes into consideration the number of questions answered, it is possible to use the formula to get OSDI values for each of the subscales [34]. Previous studies have also combined questions from different subscales to produce separate subscores using the OSDI formula [38]. Lee et al. [36] modified the OSDI questionnaire by adding questions relating to blepharitis (itchy eyes and matter along the eyelid margin), to increase the questionnaires' sensitivity to detect DF. Similar to Lee et al. [36], questions relating to itchy eyes and particulate matter along the eyelid margin were added to the current questionnaire. Questions on blurred vision and poor vision were removed from the symptom subscale and replaced with symptom questions from other validated dry eye questionnaires; McMonnies (dryness, burning), 5-item Dry Eye Questionnaire (DEQ-5) (dryness, watery), Standard Patient Evaluation of Eye Dryness (SPEED) (dryness, burning sensation, watery eyes). Novel questions (itchy eyes and red eyes) not found in previous dry eye questionnaires were included due to the high reports of such symptoms found in the literature [7, 15]. Symptoms were analysed in three different ways: the presence of symptoms, the total modified OSDI score, and the severity of symptoms. The presence of symptoms was defined as asymptomatic or symptomatic (regardless of severity). The severity of symptoms was graded from the total modified OSDI symptom score as shown in Table 1 [34, 37, 39].

\section{Questionnaire validation}

Suitability for use is dependent on the questionnaire being reliable and reproducible: that any change detected by the instrument is real and not as a result of poor repeatability [40]. The reliability and reproducibility of the questionnaire was measured in two ways. First, the internal consistency of the questionnaire was assessed using Cronbach's $\alpha$. In keeping with the literature, alpha value $>0.7$ was accepted [41, 42]. Second, the intra-rater reliability was assessed using intra-class correlation (ICC) [43] and the test-retest method; $P<0.4$ signifies poor reliability, $0.4 \leq P \geq 0.75$ signifies fair to good reliability and $P \geq 0.75$ signifies excellent reliability [44].

It is necessary that the questionnaire is also responsive and sensitive to change as a result of treatment [40]. This was measured by conducting a test-retest post-treatment for both a non-treatment

Table 1 Severity of symptoms in accordance with total modified OSDI score

\begin{tabular}{ll}
\hline Grade & Modified OSDI score \\
\hline G0: Asymptomatic & $0-12$ \\
G1: Mild & $13-22$ \\
G2: Moderate & $23-32$ \\
G3: Severe & $33-100$ \\
\hline
\end{tabular}


group and a treatment group and comparing the mean using a 2-tailed $t$ test $(P<0.05$ significance).

To allow for comparison with previous methods of validation of dry eye questionnaires, a factor analysis was applied to the symptom section of the questionnaire to determine if subscales similar to the OSDI dry eye questionnaire existed. Cronbach's $\alpha$ was applied to each subscale.

A receiver operating characteristics curve was generated to determine the sensitivity of the symptom questionnaire for the diagnosis of DF infestation at each symptom grade.

\section{Statistical analysis}

Statistical analysis was performed using the mathematical programme IBM SPSS (ver. 24.0). All summarised continuous data was expressed as mean and range. The data between categorical variables was assessed using Chi square analysis $\left(X^{2}\right)$. Betweengroup data was assessed using the Student's t test, Mann-Whitney $U$ test (MWU) and Kruskal-Wallis $\mathrm{H}$ $(\mathrm{K}-\mathrm{W})$ test where appropriate. Spearman's correlation co-efficient $\left(r_{\mathrm{s}}\right)$ was used to assess scaled and continuous variables.

\section{Results}

\section{Questionnaire validation}

One hundred and fifty-six subjects completed the questionnaire once. Suitable subjects were invited to enrol in a separate interventional DF study. Data analysis is ongoing and will be discussed in a separate paper upon completion. Fifty separate subjects completed the questionnaire twice for the test-retest assessments.

Factor analysis was applied to results from the 156 participants (age: $45.18 \pm 18.36$ years, gender: male $n=70 ; 45 \%$, female $n=86 ; 55 \%$ ) who filled out the questionnaire at least once. Extraction method 'principal axis factoring' was chosen, as the data was nonparametrically distributed [45]. Factor analysis showed three sub-scales for the questionnaire similar to the OSDI questionnaire: ocular symptoms, vision related function and environmental triggers [34] (Table 2).
Cronbach's $\alpha$ was applied to each subscale and to the questionnaire as a whole. Cronbach's $\alpha$ for the overall symptom questionnaire was good at 0.824 , each of the subscales had a slightly lower $\alpha$ value but were still $>0.7$, see Table 3 [41, 42].

Fifty separate subjects completed the questionnaire for the test-retest method to examine the reliability of the questionnaire. The questionnaire was administered twice, two weeks apart, at the same time of day, with no change to their daily routines or general circumstances between testing. The test-retest reliability of the questionnaire was determined by calculating the ICC. Results can be seen in Table 3. All scores exceeded 0.7 which is the desired criteria to be met [44].

An assumption of the test-retest reliability assessment is that a subject's condition remains stable between the first test and the retest, as no intervention took place. This is evident from the strong ICC value seen for symptom score of 0.893 . Taking this into consideration, a post hoc ICC was performed to compare the repeatability of the symptom score after two weeks of treatment in the ongoing interventional treatment study. It was expected that the correlation would be much weaker, as subjects' symptoms should have changed after receiving treatment. This hypothesis was confirmed with an ICC $=0.655<0.893$. A two-tailed $t$-test was applied to both sets of data. There was no significant difference in total symptom score in the test-retest group $(P=0.536)$ in comparison to a highly significant difference in retest total symptom score in the group that received treatment $(P=0.000)$. Treatments included were; Dr. Organic Tea Tree Face Wash, OcuSoft Plus lid scrubs and a once off treatment with BlephEx followed by nightly lid scrubs using OcuSoft Plus. The placebo effect of receiving treatment must be taken into consideration when assessing how effective treatments are at improving symptoms. However, for the purpose of assessing the questionnaires ability to measure change in subjective symptoms, the placebo effect is considered extraneous.

The receiver operator characteristic (ROC) curve was plotted to evaluate the diagnostic ability of the symptom questionnaire to assess for the presence of DF. A relatively flat ROC curve was formed, with an area under the curve (AUC) of 0.614 (Fig. 1). A positive symptom result $\geq$ G1 gives a sensitivity of $70.8 \%$ and a specificity of $46.9 \%$ for the questionnaire. 
Table 2 Factor analysis was applied to the modified OSDI questionnaire

\begin{tabular}{|c|c|c|c|}
\hline & \multicolumn{3}{|l|}{ Factor } \\
\hline & Ocular symptoms & Vision related function & Environmental triggers \\
\hline \multicolumn{4}{|l|}{ Factor analysis } \\
\hline Dryness & 0.534 & & \\
\hline Gritty/irritated & 0.696 & & \\
\hline Itchy & 0.636 & & \\
\hline Red eyes & 0.546 & & \\
\hline Burning & 0.403 & 0.373 & -0.233 \\
\hline Photophobia & & 0.342 & \\
\hline Watery & & & 0.614 \\
\hline Lids stuck together & 0.209 & & \\
\hline Reading & & 0.722 & \\
\hline Night driving & & 0.518 & \\
\hline Computer & & 0.745 & \\
\hline Television & & 0.499 & 0.244 \\
\hline Wind & & & 0.886 \\
\hline Cold air & 0.230 & & 0.610 \\
\hline Air conditioning & 0.220 & 0.246 & 0.277 \\
\hline
\end{tabular}

Extraction method: principal axis factoring

Rotation method: oblimin with Kaiser normalization

Kaiser-Meyer-Olkin measure of sampling adequacy: 0.784

Results show 3 subscales similar to OSDI; Ocular Symptoms, Vision Related Function, Environmental Triggers. Burning sensation, discomfort in cold air and discomfort in air conditioned environments loaded on more than one factor. This is likely due to the multifactorial nature of dry eye and common crossover between symptoms and causes

Table 3 Reliability analysis: Cronbach's $\alpha$ measuring internal consistency, and ICC measuring repeatability for the questionnaire

$\begin{array}{ll}\text { Internal consistency: Cronbach's } \alpha \text { (95\% } & \begin{array}{l}\text { Test-retest: Intra-class Correlation Co-efficient }(95 \% \\ \text { confidence interval) }(n=156)\end{array}\end{array}$

\begin{tabular}{lll}
\hline Reliability of the symptom questionnaire & \\
Ocular symptoms & 0.741 & 0.824 \\
Vision related function & 0.795 & 0.725 \\
Environmental triggers & 0.825 & 0.887 \\
Symptom & 0.839 & 0.897 \\
questionnaire as a & & \\
whole & &
\end{tabular}

Results are shown for each of the subscales and for the overall questionnaire as a whole. All values were $>0.7$

As expected, as severity of symptoms increase (G3 severe symptoms), the sensitivity drops to $33 \%$, but the specificity increases to $85.7 \%$.

\section{Questionnaire application}

One hundred and fifty-six subjects completed the questionnaire and were assessed for presence and quantity of DF. The overall prevalence of DF found was $68 \%$. The total mean number of DF found per subject on microscopic examination was 3.83 mites (range 0-25). Subjects included 70 males and 86 females with a mean age of $45.98 \pm 18.36$ years (range 19-82 years). The presence and quantity of DF was not significantly different between genders ( $P=0.061$ and $P=0.695$, respectively). Females, 


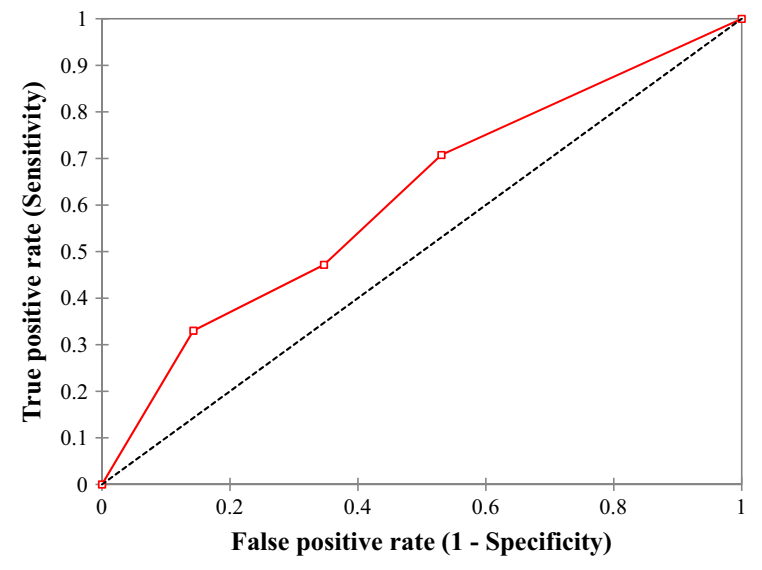

Fig. 1 ROC curve demonstrates the ability of the novel questionnaire to assess for presence of DF using symptom grade (Normal-Severe: $0-3$ ). AUC $=0.614$

however, were more symptomatic $(P=0.026)$ (Table 4). The presence of DF was significantly associated with increasing age ( $t$-test; $P=<0.001$ ). Likewise, the quantity of DF was significantly associated with increasing age also $\left(\mathrm{r}_{\mathrm{s}}\right.$ 0.386; $P=<0.001)$.

Presence of Demodex folliculorum versus symptoms

Subjects with DF were more symptomatic than those without DF ( $X^{2} ; P=0.04$ ) (Fig. 2). As such, the total modified symptom score was significantly higher in subjects with DF $(25.01 \pm 18.86)$ in comparison to subjects without DF $(18.81 \pm 15.55) \quad$ (MWU; $P=0.05)$. Likewise, the severity of symptoms increased in subjects with DF in comparison to subjects without DF $\left(X^{2} ; P=0.028\right)$ (Fig. 3). The greatest difference was found in the severe symptom group. Very few $(n=7)$ subjects without DF had severe symptoms (Fig. 3). The majority of subjects without DF were asymptomatic. By comparison, the majority of subjects with DF had symptoms; the

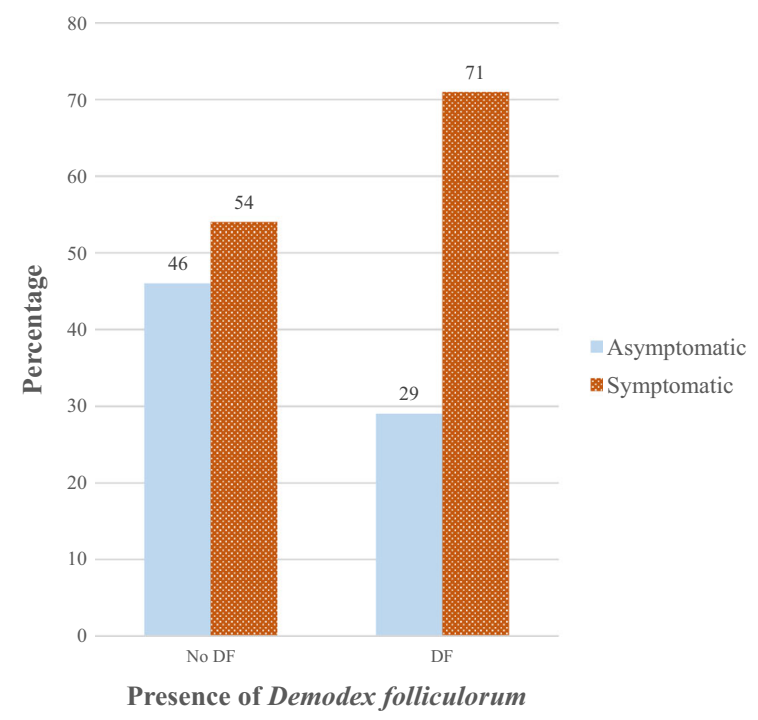

Fig. 2 Percentage frequency distribution of symptomatic and asymptomatic subjects, with and without DF. Subjects with DF were significantly more symptomatic $\left(X^{2} ; P=0.04\right)$

majority of which were severely symptomatic $(n=35)$ (Fig. 3).

The prevalence of each symptom reported by subjects with and without DF is shown in Table 5. The presence of the symptom itch was significantly associated with the presence of DF. A symptom of 'itchy eyes' was more commonly reported by subjects with DF than those without (69 vs. 52\%) $\left(X^{2}\right.$; $P=0.025)$. The severity of itch for the presence of DF was not found to be significant $\left(X^{2} ; P=0.131\right)$. Overall subjects with 'itchy eyes' were not significantly older than those without 'itchy eyes' (MWU; $P=0.826)$. Subject's with 'itchy eyes' and DF were significantly older than those with 'itchy eyes' and no DF (MWU; $P=<0001$ ). Asymptomatic individuals with DF were also older, but not significantly (MWU; $P=0.323$ ).

A functional vision symptom of 'discomfort watching television' was also significantly associated with

Table 4 Comparison of age, presence and quantity of DF, and OSDI scores for male and female study participants'

\begin{tabular}{llllll}
\hline Sex & $N$ & Age (years) & Prevalence $(\%)$ & Demodex count & OSDI score \\
\hline Male & 70 & $44.83 \pm 18.03$ & 74.29 & 4.02 (range 0-22) & $19.54 \pm 15.69$ \\
Female & 86 & $46.93 \pm 18.69$ & 62.79 & 3.69 (range 0-25) & $25.87 \pm 19.39$ \\
& $P=0.477(\mathrm{~A})$ & $P=0.126(\mathrm{~B})$ & $P=0.695(\mathrm{~A})$ & $P=0.026(\mathrm{~A})$ & \\
\hline
\end{tabular}




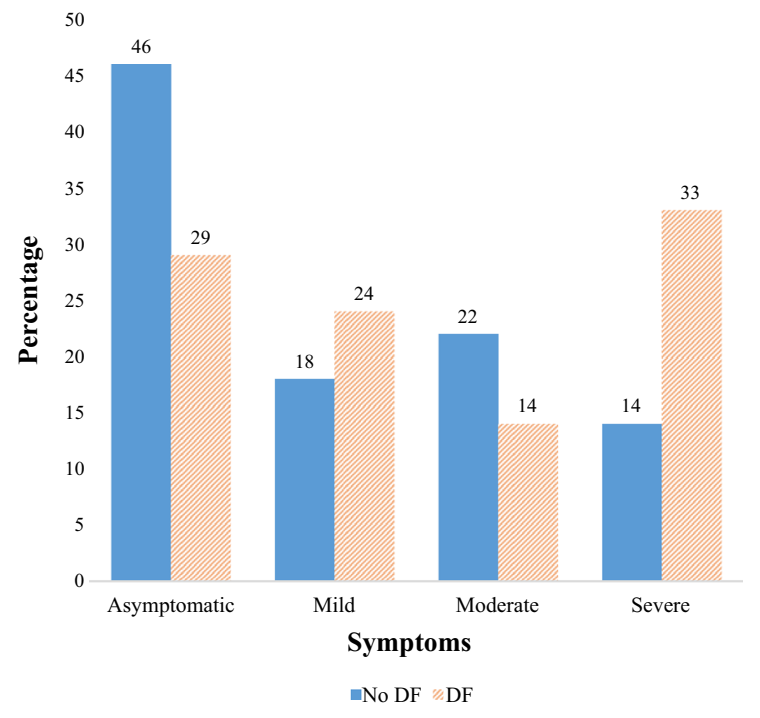

Fig. 3 Percentage frequency distribution of grade of severity of symptoms among subjects with and without DF $\left(X^{2}\right.$; $P=0.028) .46 \%$ of subjects without DF were asymptomatic and only $14 \%$ had severe symptoms. Only $29 \%$ of subjects with DF were asymptomatic, however, $33 \%$ had severe symptoms. It is also evident that the majority of symptomatic subjects with DF were severely symptomatic

the presence of DF (42 vs. $12 \%)\left(X^{2} ; P=0.000\right)$. However, overall subjects reporting 'discomfort when watching television' were significantly older than those without any discomfort (MWU; $P=0.010$ ). As the symptomatic group is older, they will be predisposed to an increase in presence of DF given the significant risk factor that age is for DF $[15,46]$.

\section{Quantity of Demodex folliculorum versus symptoms}

The total mean number of DF found per subject on microscopic examination was 3.83 mites (range $0-25$ ). Not all subjects with DF were symptomatic. The mean number of mites found was significantly higher among symptomatic subjects (4.53 mites: range $0-25$ ) in comparison to asymptomatic subjects (2.51 mites: range 0-19) (MWU; $P=0.021$ ). Spearman's correlation showed no significant relationship between quantity of DF and the modified OSDI score $\left(r_{\mathrm{s}}=0.122 ; P=0.128\right)$. However, a low positive correlation was established between the number of DF mites and increasing grade of severity of symptoms $\left(r_{\mathrm{s}}=0.162 ; \quad P=0.0439\right)$. This correlation is expressed in the equation: $Y=0.0267 X$ (number of $\mathrm{DF})+1.2565$, and suggests that on average $1 \mathrm{DF}$ mite $=$ G1.28 symptoms. An increase of 28 more mites is required to produce moderate symptoms, and a further 38 extra (minimum 66 mites) required to produce severe symptoms. Spearman's correlation demonstrated an equation similar to above, $Y=0.0264 X$ (number of DF) +1.0552 , that an increase in severity of 'itchy eyes' was also positively associated with an increase in the quantity of DF

Table 5 Prevalence of symptoms reported by subjects with and without DF; given as percentage of subjects in each group

\begin{tabular}{llll}
\hline Symptom & Subjects with Demodex folliculorum $(\%)$ & Subjects without & Demodex folliculorum $(\%)$ \\
\hline Gritty/irritated & 72 & 70 & $P$ value $\left(X^{2}\right)$ \\
Itchy & 69 & 52.0 & 0.685 \\
Dryness & 68 & 68 & 0.025 \\
Wind & 57 & 62 & 0.880 \\
Air conditioning & 55 & 44 & 0.459 \\
Watery & 53 & 60 & 0.251 \\
Photophobia & 45 & 46 & 0.347 \\
Red eyes & 45 & 40 & 0.959 \\
Computer & 43 & 40 & 0.378 \\
Cold air & 43 & 30 & 0.656 \\
Problems reading & 42 & 36 & 0.167 \\
Television & 42 & 12 & 0.300 \\
Burning sensation & 28 & 18 & 0.000 \\
Lids stuck together & 21 & 12 & 0.137 \\
Night driving & 19 & 8 & 0.184 \\
\hline
\end{tabular}


$\left(r_{\mathrm{s}}=0.172 ; P=0.035\right)$. Likewise, this equation suggests that $1 \mathrm{DF}=\mathrm{G1} .08$ ('itchy eyes' some of the time), but on average, an increase of a further 36 DF mites, respectively, are required to produce respective increases in severity of symptoms. Thus, the above equations for severity of symptoms and severity of itch show that the presence of just $1 \mathrm{DF}$ can produce mild symptoms, but symptoms can also be present without DF, as seen previously.

An increase in severity of 'discomfort when watching television' was found to be significantly associated with an increase in quantity of DF $\left(r_{\mathrm{s}}=0.164 ; P=0.044\right)$. Further analysis showed that this increase was directly parallel to an increasing age for the same symptom $\left(r_{\mathrm{s}}=0.24 ; P=0.003\right)$. Given the low overall prevalence $(32 \%)$ of 'discomfort when watching television', even among subjects with DF, and the significant increase in age of those symptomatic, it is possible that age-related dry eye changes may be contributing to this symptom. However, presence and quantity of DF should still be considered as it is an age-related change, and very few control subjects reported discomfort.

Time of day did not appear to affect symptoms with respect to DF. No other individual symptom was found to be significantly associated with the presence or quantity of DF.

\section{Pathogenic infestation}

Earlier studies have suggested not all incidence of DF is pathogenic, and it appears to be an increase in quantity of DF that causes "pathogenic" infestation $[47,48]$. Using the severity scale suggested by Randon et al. [48], $\geq 3$ mites per follicle, data was analysed to look at the prevalence of "pathogenic" DF in the study population, and any associations between "pathogenic" DF infestation and age and symptoms (Table 6).

Subjects with "pathogenic" DF infestation were significantly older than subjects with "non-pathogenic" DF infestation (MWU; $P=0.014$ ) and subjects with no DF (MWU; $P<0.001$ ). Subjects with "non-pathogenic" DF infestation were older, but not significantly, than subjects with no DF (MWU; $P=0.067$ ).

Subjects with "pathogenic" and "non-pathogenic" DF were more symptomatic than subjects with no DF, although this difference was not found to be significant for either group (MWU; $P=0.076$ and $P=0.118$, respectively). The highest prevalence of the symptom 'itchy eyes' was among subjects with "pathogenic" DF, however, when compared to subjects with no DF, this was not significant $\left(X^{2} ; P=0.077\right)$.

\section{Discussion}

Demodex folliculorum is commonly associated with ocular surface disease [7]. The prevalence of DF reported in the literature varies from 20 to $100 \%$, increasing with age and existing presence of ocular surface disease. Kemal et al. [49] found a prevalence of $28.8 \%$ in blepharitis subjects and $26.7 \%$ among normal individuals. Gao et al. [11] reported a prevalence of $100 \%$ among patients with CD, and $22 \%$ among patients without CD. Kabataş et al. [22] discovered a prevalence of $67.2 \%$ in blepharitis patients and $54.9 \%$ in control patients. Roth [46] reported an overall prevalence of $84 \%$ that increased to $100 \%$ in subjects over 70 years of age. Lee et al. [36] found an overall prevalence of $70 \%$ in their demographic epidemiology study. The present study, in good agreement with previous studies, found an overall prevalence of $68 \%$ which was higher in older and in symptomatic subjects.

The current study discovered a relatively high prevalence of DF, even among normal population. However, there is an ongoing debate over the pathogenicity of the ectoparasites [7, 50-52]. The findings in this study are in agreement with previous authors [53, 54], that DF can be found among asymptomatic individuals. The present study found an overall mean number of 2.51 mites (range 0-19) among asymptomatic individuals. Lacey et al. [52] suggested that in many cases DF is a mere commensal organism with possible beneficial roles-ingesting bacteria and other micro-organisms in the lash follicle. Forton et al. [55] demonstrated in dermatology that $<5 \mathrm{mites} / \mathrm{cm}^{2}$ is considered normal, and that higher densities are seen in the presence of disease or immunodeficiency. Baima et al. [50] postulated that the mite has the potential to become pathogenic to the host where numbers of mites increase beyond a critical level. Randon et al. [48] suggest this level as $\geq 3$ mites per follicle. The increase in symptoms, as seen in previous studies [15, 22, 23, 36] and in the current study also, would suggest that DF does have 
Table 6 Comparison of quantity of DF, age, presence of symptoms, modified OSDI score, and presence of itch for subjects with; no $\mathrm{DF}$, mild "non-pathogenic" infestation, and "pathogenic" infestation of DF. A Kruskal-Wallis $P$ value: $\mathrm{B}=X^{2} P$ value

\begin{tabular}{|c|c|c|c|c|c|c|}
\hline & $\begin{array}{l}\text { Frequency } \\
(n) \text { (Prevalence } \\
\%)\end{array}$ & $\begin{array}{l}\text { Quantity of } \\
\text { mites } \\
\text { (mean } \pm \text { SD) }\end{array}$ & $\begin{array}{l}\text { Age (yrs) } \\
(\text { mean } \pm S D)\end{array}$ & $\begin{array}{l}\text { Presence of } \\
\text { Symptoms } \\
(\%)\end{array}$ & $\begin{array}{l}\text { Modified } \\
\text { OSDI } \\
(\text { mean } \pm \text { SD) }\end{array}$ & $\begin{array}{l}\text { Presence } \\
\text { of Itch }(\%)\end{array}$ \\
\hline No Demodex folliculorum & $50(32 \%)$ & $0.00 \pm 0.00$ & $37.76 \pm 17.02$ & 54.00 & $18.81 \pm 15.55$ & 52.00 \\
\hline $\begin{array}{l}\text { Mild/Normal infestation }(<3 \\
\text { mites per follicle) }\end{array}$ & $39(25 \%)$ & $1.54 \pm 0.68$ & $44.62 \pm 19.07$ & 64.10 & $25.79 \pm 19.49$ & 66.67 \\
\hline $\begin{array}{l}\text { Pathogenic infestation }(\geq 3 \\
\text { mites per follicle) }\end{array}$ & $60(38.5 \%)$ & $8.97 \pm 5.20$ & $53.60 \pm 16.51$ & 75.00 & $24.02 \pm 17.33$ & 71.67 \\
\hline \multirow{2}{*}{$\begin{array}{l}\text { Mites visible on lash } \\
\text { manipulation but not on } \\
\text { microscope }\end{array}$} & $7(4.5 \%)$ & $0.00 \pm 0.00$ & $47.14 \pm 14.36$ & 71.43 & $29.27 \pm 28.79$ & 57.14 \\
\hline & & & $<0.001(\mathrm{~A})$ & $0.141(\mathrm{~B})$ & $0.274(\mathrm{~A})$ & $0.161(\mathrm{~B})$ \\
\hline
\end{tabular}

pathogenic potential as the numbers of DF present an increase. Furthermore, in an ongoing study, these symptoms were shown to be improving following treatment, thus strengthening the argument that the DF mites do have pathogenic potential. Sędzikowska et al. [23] recently published results of a large-scale study investigating the association between DF and presence of symptoms reported by patients without the use of a questionnaire. The results propose that a minimum of $7 \mathrm{DF}$ mites per 8 epilated eyelashes is required to produce 1 symptom, and that a further 18 mites are required to produce a second symptom. In the study conducted by Sędzikowska et al. [23], they did not measure severity of these symptoms reported by patients: intermittent vs constant, sometimes vs all of the time, merely the presence of the symptom. The current study investigated the severity of symptoms reported by patients using a modified version of the OSDI questionnaire. Applying a similar statistical analysis as that applied by Sędzikowska et al. [23], the current study found a much higher number (33) of DF was required to produce moderate symptoms. While the results of both studies show good agreement, they are not directly comparable, as the current study counted DF mites on 4 epilated eyelashes, whereas Sędzikowska et al. [23] counted DF mites on 8 epilated eyelashes.

Symptoms can be present in the absence of DF, and similarly DF can be present in the absence of symptoms. A prevalence of $68 \%$ DF was found in the current study. Of these affected individuals, only $71 \%(75 / 106)$ had symptoms, $\left(X^{2} ; \quad P=0.028\right)$
(Fig. 2). A potential reason for this is that chronic inflammation of the anterior ocular surface can lead to changes in corneal morphology [56], leading to corneal hypoesthesia, thus resulting in reduced symptoms in the presence of severe infestation and inflammation [57-59]. As a result, patient reported symptoms do not always correlate well with clinical signs of ocular surface disease, as was demonstrated in the current study. While it appears that the majority of individuals with DF have symptoms; nonetheless, asymptomatic individuals can also have DF. As such, this study found that among those 106 individuals with DF, the quantity of DF seen on microscopic examination was similar between asymptomatic and symptomatic subjects $(4.39 \pm 4.37$ mites vs $6.16 \pm 5.82$ mites, respectively; MWU; $P=0.185$ ). A potential cause for this could be that chronic infestation and inflammation has resulted in hypoesthesia at the ocular surface resulting in an asymptomatic subject with large numbers of DF. However, as DF reside in the follicle, the crawling, scrapping movement of the mites would be expected to create an itching sensation, which should not be affected by corneal hypoesthesia. A more likely possibility is that not all DF was removed during lash epilation. During the study, it became apparent that especially in cases where lashes were loose in damaged lash follicles due to the presence of high numbers of DF, the lash would slip out leaving the DF still inside the lash follicle. This further enhances the need to combine symptoms and a thorough clinical work-up when screening and treating patients for DF. It should be taken into consideration 
when assessing for the presence of DF that as the severity of infestation increases, the looser the lashes become. This increases the possibility of DF mites remaining in the lash follicles. The authors recommend using lash manipulation in combination with epilation to accurately assess the severity of DF infestation. Further research into the benefit of lash manipulation is currently taking place and will be described in more detail in a future paper.

The literature reports certain symptoms commonly associated with DF; itch, burning sensation, foreign body sensation, redness and crusts along the lid margins, blurred vision and misdirection of eyelashes [7, 15, 22, 23, 60-62]. Ocular dryness (74.7\%), itching $(42.78 \%)$, and irritation $(39.1 \%)$ were reported in a study by Koo et al. [15]. Kabataş et al. [22] stated prevalence values of symptoms of redness $(80 \%)$, itching $(63.6 \%)$ and foreign body sensation $(55.6 \%)$ in DF positive blepharitis patients. Sędzikowska et al. [23] reported similar symptoms but lower values: itching (28\%), redness (21\%), watery eyes (15\%), and dryness $(6 \%)$ among others. The use of specific questionnaires was not discussed by Kabataş et al. [22] or Sędzikowska et al. [23]. The lower prevalence values reported in the study by Sędzikowska et al. [23] are likely as a result of symptom reporting not being prompted by the use of a questionnaire; but depended on each subject complaining of a symptom of their own accord. The most commonly reported symptoms by subjects with DF in the current study were gritty irritated eyes (72\%) followed by; itch (69\%), dryness $(68 \%)$, watery $(57 \%)$, photophobia $(45 \%)$, red eyes (45\%), burning sensation (28\%), and lids stuck together $(21 \%)$. Many of these symptoms were also commonly reported by subjects that did not have any $\mathrm{DF}$, and were not found to be significantly associated with DF. However, in agreement with previous studies $[22,23,62]$, the current study found that the symptom 'itchy eyes' was associated with an increased presence of DF. Furthermore, the current study demonstrated that the severity of 'itchy eyes' increased as the number of DF increased. This further strengthens the basis for 'itchy eyes' as a significant symptom of DF infestation.

It should be noted that the symptom 'itchy eyes' was also reported by approximately $50 \%$ of the control subjects, this was not unexpected. Dry eye is multifactorial and its symptoms are subjective, and therefore can be interpreted differently by each individual.
For example, itch is one of the hallmark symptoms of allergy. Data collection for the current study took place over 2 years, therefore, it is possible that a history of allergy influenced the severity of the symptom itch. However, Chi square analysis showed no significant correlation between the presence of allergy and the presence of general symptoms, or symptoms of itch among subjects that did not have DF ( $X^{2} ; P=0.79$ and $P=0.085$, respectively). The authors are not suggesting 'itchy eyes' as a diagnostic symptom of DF; merely that it appears to be a more prevalent and frequently reported symptom among individuals affected with DF infestation. Potentially, the presence of the symptom itch is as an allergic response to the presence of DF which has been hypothesised previously [7, 63].

The OSDI questionnaire is one of the most commonly used symptom questionnaires administered to subjects in DF related clinical trials [15, 35, 36]. Results from the present study and previous studies outlined above have demonstrated that itch is amongst the most common complaint in subjects with DF. However, no question on the original OSDI exists to ask about 'itchy eyes'. The modified questionnaire developed by Lee et al. [36] included a question on 'itchy eyes', and demonstrated that the overall OSDI score was significantly associated with increased numbers of DF. Nonetheless, the authors of the current study were unable to infer if the questionnaire modified and administered by Lee et al. [36] had been validated and therefore would suggest the utilisation of the current questionnaire, or similar validated questionnaire (that includes questions regarding itchy eyes) in future studies relating to DF.

\section{Conclusion}

The novel questionnaire demonstrated good internal consistency (Cronbach's $\alpha$ was $>0.7$ for both the total questionnaire and each of the subscales) and good to very good repeatability $(>0.75)$ for both the total questionnaire and each of the subscales in the testretest ICC. The strong repeatability component of the questionnaire shows that it can be used as a valid means of monitoring patient's subjective symptoms as a result of treatment over time in a clinical setting. This is becoming increasingly more important as 
PROs become a critical part of patient-centred management in the health sector [26].

The questionnaire displayed a reasonable sensitivity value of $70.8 \%$ for correctly identifying subjects found positive for DF infestation. However, for verifying the presence of DF infestation and knowing who requires further intervention, this would not be sufficient and indicates that a thorough clinical workup would also be required to confirm diagnosis.

To the best of the authors' knowledge, this is the first study that has looked at the prevalence of ocular DF and its associated symptoms in an Irish population. There was a strong link between the presence and quantity of DF and severity of symptoms, using the modified OSDI questionnaire. Itch was significantly associated with the presence of DF above any other symptom reported by patients. In clinical practice, it is important to remember the presence of DF in patients complaining of itchy eyes. Based on the findings of the current study, it would be advisable to utilise the current questionnaire or a questionnaire that includes questions about itch when managing and treating anterior ocular disorders such as blepharitis. However, a thorough clinical work-up is still required for differential diagnosis between various anterior ocular disorders.

Not all patients with DF will be symptomatic, even when infestation is seemingly severe. As with many anterior ocular disorders, symptoms can be similar and may not always be present. The relationship between DF infestation and corneal hypoesthesia needs to be investigated further and additional research into the triggers that cause a patient to become symptomatic is still required.

Acknowledgements The authors' thanks the management and staff of the National Optometry Centre for providing use of the premises and equipment, and helping with diary management and subject recruitment. The final year Optometry students assisted with data collection and subject recruitment.

\section{Compliance with ethical standards}

Conflict of interest The authors declare that they have no conflict of interest. This research was conducted as part of a selffunded postgraduate research degree.

Human and animal rights All procedures performed in this study involving human participants were in accordance with the ethical standards of Dublin Institute of Technology and with the Tenets of Helsinki Declaration of Human Studies [29].
Informed consent Informed consent was obtained from all individual participants included in the study.

\section{References}

1. Spickett SG (1961) Studies on Demodex folliculorum Simon (1842). I. Life history. Parasitology 51:181-192

2. Rufli T, Mumcuoglu Y (1981) The hair follicle mites $D e$ modex folliculorum and Demodex brevis: biology and medical importance. Dermatologica 162:1-11

3. English FP, Nutting WB (1981) Demodicosis of ophthalmic concern. Am J Ophthalmol 91:362-372

4. Aylesworth R, Vance JC (1982) Demodex folliculorum and Demodex brevis in cutaneous biopsies. J Am Acad Dermatol 7:583-589. https://doi.org/10.1016/S0190-9622(82)701379

5. Lacey N, Kavanagh K, Tseng S (2009) Under the lash: Demodex mites in human diseases. Biochem (Lond) 31:2-6

6. Coston T (1967) Demodex folliculorum blepharitis. Trans Am Ophthalmol Soc 65:361-392

7. Liu J, Sheha H, Tseng SCG (2010) Pathogenic role of Demodex mites in blepharitis. Curr Opin Allergy Clin Immunol 10:505-510

8. Schear MJ, Milman T, Steiner T et al (2015) The association of Demodex with chalazia: a histopathologic study of the eyelid. Ophthalmic Plast Reconstr Surg 32:275-278. https:// doi.org/10.1097/IOP.0000000000000500

9. English F (1971) Demodex folliculorum and oedema of the eyelash. Br J Ophthal 55:742-746

10. Nicholls SG, Oakley CL, Tan A, Vote BJ (2017) Demodex species in human ocular disease: new clinicopathological aspects. Int Ophthalmol 37:303-312. https://doi.org/10. 1007/s10792-016-0249-9

11. Gao YY, Di Pascuale MA, Li W et al (2005) High prevalence of Demodex in eyelashes with cylindrical dandruff. Investig Ophthalmol Vis Sci 46:3089-3094

12. Solomon A, Dursun D, Liu Z et al (2001) Pro- and antiinflammatory forms of interleukin-1 in the tear fluid and conjunctiva of patients with dry-eye disease. Invest Ophthalmol Vis Sci 42:2283-2292

13. De Paiva C, Pflugfelder S (2008) Rationale for anti-inflammatory therapy in dry eye syndrome. Arq Bras Oftalmol 71:89-95

14. Savini G, Prabhawasat P, Kojima T et al (2008) The challenge of dry eye diagnosis. Clin Ophthalmol 2:31-55

15. Koo H, Kim TH, Kim KW et al (2012) Ocular surface discomfort and Demodex: effect of tea tree oil eyelid scrub in Demodex blepharitis. J Korean Med Sci 27:1574-1579

16. Sullivan BD, Crews L, Messmer EM et al (2014) Correlations between commonly used objective signs and symptoms for the diagnosis of dry eye disease: clinical implications. Acta Ophthalmol 92:161-166

17. Nichols K, Nichols J, Mitchell G (2004) The lack of association between signs and symptoms in patients with dry eye disease. Cornea 23:762-770

18. Sullivan BDBD, Whitmer D, Nichols KKK et al (2010) An objective approach to dry eye disease severity. Investig 
Ophthalmol Vis Sci 51:6125-6130. https://doi.org/10.1167/ iovs. $10-5390$

19. Pult H, Purslow C, Murphy PJ (2011) The relationship between clinical signs and dry eye symptoms. Eye (Lond) 25:502-510. https://doi.org/10.1038/eye.2010.228

20. Achtsidis V, Kozanidou E, Bournas P et al (2014) Dry eye and clinical disease of tear film, diagnosis and management. Eur Ophthalmic Rev 8:17-22. https://doi.org/10.17925/ EOR.2014.08.01.17

21. Kim JT, Lee H, Chun YS, Kim JC (2011) Tear cytokines and chemokines in patients with Demodex blepharitis. Cytokine 53:94-99

22. Kabataş N, Doğan AŞ, Kabataş EU et al (2017) The effect of Demodex infestation on blepharitis and the ocular symptoms. Eye Contact Lens 43:64-67

23. Sędzikowska A, Osęka M, Grytner-Zięcina B (2016) Ocular symptoms reported by patients infested with Demodex mites. Acta Parasitol 61:808-814

24. Jarmuda S, O'Reilly N, Zaba R et al (2012) Potential role of Demodex mites and bacteria in the induction of rosacea. J Med Microbiol 61:1504-1510

25. Sahai Malik P, Sahai A, Malik P (2005) Dry eye: prevalence and attributable risk factors in a hospital-based population. Indian J Ophthalmol 53:87-91. https://doi.org/10.4103/ 0301-4738.16170

26. Pesudovs K, Burr JM, Harley C, Elliott DB (2007) The development, assessment, and selection of questionnaires. Optom Vis Sci 84:663-674

27. Willke RJ (2008) Measuring the value of treatment to patients: patient-reported outcomes in drug development. Am Heal Drug Benefits 1:34-40

28. Schaumberg DA, Nichols JJ, Papas EB et al (2011) The international workshop on meibomian gland dysfunction: report of the subcommittee on the epidemiology of, and associated risk factors for, MGD. Investig Ophthalmol Vis Sci 52:1994-2005

29. World Medical Association (2013) World Medical Association declaration of Helsinki ethical principles for medical research involving human subjects. JAMA 310:2191-2194

30. Gao YY, Di Pascuale MA, Li W et al (2005) In vitro and in vivo killing of ocular Demodex by tea tree oil. $\mathrm{Br} \mathrm{J}$ Ophthalmol 89:1468-1473. https://doi.org/10.1136/bjo. 2005.072363

31. Mastrota KM (2013) Method to identify Demodex in the eyelash follicle without epilation. Optom Vis Sci 90:e172e174. https://doi.org/10.1097/OPX.0b013e318294c2c0

32. Wolffsohn JS, Arita R, Chalmers R et al (2017) TFOS DEWS II diagnostic methodology report. Ocul Surf 15:539-574. https://doi.org/10.1016/j.jtos.2017.05.001

33. Tomlinson A, Bron AJ, Korb DR et al (2011) The international workshop on meibomian gland dysfunction: report of the diagnosis subcommittee. Investig Ophthalmol Vis Sci 52:2006-2049

34. Schiffman RM, Christianson MD, Jacobsen G et al (2000) Reliability and validity of the ocular surface disease index. Arch Ophthalmol 118:615. https://doi.org/10.1001/ archopht.118.5.615

35. Jalbert I, Rejab S (2015) Increased numbers of demodex in contact lens wearers. Optom Vis Sci 92:671-678
36. Lee SH, Chun YS, Kim JH et al (2010) The relationship between demodex and ocular discomfort. Investig Ophthalmol Vis Sci 51:2906-2911

37. Walt J (2004) Ocular surface disease index (OSDI) administration and scoring manual

38. Mathews PM, Ramulu PY, Friedman DS et al (2013) Evaluation of ocular surface disease in patients with glaucoma. Ophthalmology 120:2241-2248. https://doi.org/10. 1016/j.ophtha.2013.03.045

39. Miller KL, Walt JG, Mink DR et al (2010) Minimal clinically important difference for the ocular surface disease index. Arch Ophthalmol 128:94-101. https://doi.org/10. 1001/archophthalmol.2009.356 (Chicago, Ill 1960)

40. Smith JA, Albenz J, Begley C et al (2007) The epidemiology of dry eye disease: report of the epidemiology subcommittee of the international dry eye workshop (2007). Ocul Surf 5:93-107

41. Bland JM, Altman DG (1997) Statistics notes: Cronbach's alpha. BMJ. https://doi.org/10.1136/bmj.314.7080.572

42. Tavakol M, Dennick R (2011) Making sense of Cronbach's alpha. Int J Med Educ 2:53-55

43. Hallgren KA (2012) Computing inter-rater reliability for observational data: an overview and tutorial. Tutor Quant Methods Psychol 8:23-34

44. Rosner B (2011) Fundamentals of biostatistics, 7th edn. Brooks/Cole, Boston

45. Costello AB, Osborne JW, Costello AB (2009) Best practices in exploratory factor analysis: four recommendations for getting the most from your analysis. Pan Pacific Manag Rev 12:131-146

46. Roth AM (1979) Demodex folliculorum in hair follicles of eyelid skin. Ann Ophthalmol 11:37-40

47. Forton F, Seys B (1993) Density of Demodex folliculorum in rosacea: a case-control study using standardized skin-surface biopsy. Br J Dermatol 128:650-659

48. Randon M, Liang H, El Hamdaoui M et al (2015) In vivo confocal microscopy as a novel and reliable tool for the diagnosis of Demodex eyelid infestation. Br J Ophthalmol 99:336-341. https://doi.org/10.1136/bjophthalmol-2014305671

49. Kemal M, Sümer Z, Toker MI et al (2005) The prevalence of Demodex folliculorum in blepharitis patients and the normal population. Ophthalmic Epidemiol 12:287-290

50. Baima B, Sticherling M (2002) Demodicidosis revisited. Acta Derm Venereol 82:3-6

51. Elston DM (2010) Demodex mites: facts and controversies. Clin Dermatol 28:502-504

52. Lacey N, Ní Raghallaigh S, Powell FC (2011) Demodex mites-commensals, parasites or mutualistic organisms? Dermatology 222:128-130

53. de Venecia AB, Lim Bon Siong R (2011) Demodex sp. infestation in anterior blepharitis, meibomian-gland dysfunction, and mixed blepharitis. Philipp J Ophthalmol $36: 15-22$

54. Bhandari V, Reddy JK (2014) Blepharitis: always remember demodex. Middle East Afr J Ophthalmol 21:317-320

55. Forton F, Germaux M-A, Brasseur T et al (2005) Demodicosis and rosacea: epidemiology and significance in daily dermatologic practice. J Am Acad Dermatol 52:74-87 
56. Villani E, Magnani F, Viola F et al (2013) In vivo confocal evaluation of the ocular surface morpho-functional unit in dry eye. Optom Vis Sci 90:576-586

57. Hoşal BM, Örnek N, Zilelioğlu G, Elhan AH (2005) Morphology of corneal nerves and corneal sensation in dry eye: a preliminary study. Eye 19:1276-1279

58. Bourcier T, Acosta MC, Borderie V et al (2005) Decreased corneal sensitivity in patients with dry eye. Investig Ophthalmol Vis Sci 46:2341

59. Lemp MA, Baudouin C, Baum J et al (2007) The definition and classification of dry eye disease: report of the definition and classification subcommittee of the international dry eye work shop (2007). Ocul Surf 55:75-92
60. Gao YY, Di Pascuale MA, Elizondo A, Tseng SC (2007) Clinical treatment of ocular demodicosis by lid scrub with tea tree oil. Cornea 26:136-143

61. Kheirkhah A, Casas V, Li W et al (2007) Corneal manifestations of ocular Demodex infestation. Am J Ophthalmol 143:743-749

62. Kojima T, Ishida R, Sato EA et al (2011) In vivo evaluation of ocular demodicosis using laser scanning confocal microscopy. Investig Ophthalmol Vis Sci 52:565-569

63. Georgala S, Katoulis AC, Kylafis GD et al (2001) Increased density of Demodex folliculorum and evidence of delayed hypersensitivity reaction in subjects with papulopustular rosacea. J Eur Acad Dermatol Venereol 15:441-444 